\title{
GALOIS THEORY FOR CYLINDRIC ALGEBRAS AND ITS APPLICATIONS
}

BY

\author{
STEPHEN D. COMER
}

\begin{abstract}
A Galois correspondence between cylindric set algebras and permutation groups is presented in this paper. Moreover, the Galois connection is used to help establish two important algebraic properties for certain classes of finite-dimensional cylindric algebras, namely the amalgamation property and the property that epimorphisms are surjective.
\end{abstract}

The importance of the amalgamation property (AP for short) in algebraic logic has been recognized for a long time. In [16] the connection with the interpolation property of first-order logic is discussed. The positive amalgamation results from the author's thesis [2] and their extensions announced in [3] are cited in \$2.4 of [16]. These results are established, with a slight improvement, in $\$ 4$ below. The key to this study is a lattice anti-isomorphism between subgroups of the symmetric group $S_{\mu}$ and subalgebras of the full set algebra $\mathfrak{A}(\alpha, \mu)$ when $0<\mu \leqslant \alpha+1<\omega$. This Galois theory is developed in $\$ 2$. It is applied in $\$ 3$ to show that the algebra $\mathfrak{A}(\alpha, \mu)$, $0<\mu \leqslant \alpha+1<\omega$, is homogeneous and the variety it generates has enough injectives. The announcement [3] dealt with the case $\mu \leqslant \alpha$. In this treatment of Galois theory we also include the results of $H$. Andréka and I. Németi which show that the theory works for $\mu=\alpha+1$, but for no larger $\mu$.

In $\$ 5$ the property (ES) that all epimorphisms are surjective is established for certain varieties of $\mathrm{CA}_{\alpha}$ 's. It has been shown by I. Németi (see [14]) that the ES property for classes of $\mathrm{CA}_{\alpha}$ 's is related to Beth's definability result. The question of when the ES property holds was raised in [8, p. 311, Problem 10].

To put the results in this paper in perspective, perhaps an additional remark will be useful. Many of the results assert that some property (that depends on $\alpha$ and $\mu$ ) holds if $\mu \leqslant \alpha+1<\omega$. Upon reading a preliminary draft of the paper, Andréka and Németi showed that most of the main results could not be improved in the sense that examples show that the properties fail for $\omega>\mu>\alpha+1$. In particular. this applies to Theorems 2.2, 2.8, 2.9, 3.7, 3.8(2), Corollaries 3.10, 5.5 and Lemma 5.2(2). Essentially the only result where the value of $\mu$ is not known to be the best possible is in Theorem 2.5.

Received by the editors July 6, 1983. Some of the contents of this paper were presented at the 74th Annual meeting of the American Mathematical Society in San Francisco. California, January 23-27, 1968.

1980 Mathematics Subject Classification. Primary 03G15; Secondary 06A15, 08 B25.

Key words and phrases. Cylindric algebras, Galois correspondence, amalgamation property, epimorphisms, injectives.

'Research supported by NSF grant MCS-8003896 and by The Citadel Development Foundation. 
The author wishes to thank $H$. Andréka and I. Németi for their generous hospitality, stimulating discussions and correspondence concerning properties of set algebras. It was also due to their encouragement that the unpublished portions of [2] and [3] are at last, being made available.

1. Preliminaries. We primarily use the notation and terminology of [7 and 8], but in order to make the paper reasonably self-contained a quick summary of basic terminology and some unpublished results is given in this section.

The class of all cylindric algebras of dimension $\alpha$ is denoted $\mathrm{CA}_{\alpha}$ and the class of all polyadic equality algebras by $\operatorname{PEA}_{\alpha}$. The collection $\operatorname{Sb}\left({ }^{\alpha} U\right)$ of all subsets of ${ }^{\alpha} U$ can be made into a cylindric field of sets in a natural way. This algebra is referred to as the full set algebra with base $U$ and denoted by $\mathfrak{A}(\alpha, U)$. Subalgebras of full set algebras are called set algebras and the class of all such $\mathrm{CA}_{\alpha}$ 's is denoted $\mathrm{Cs}_{\alpha}$. The class of all $\mathrm{Cs}_{\alpha}$ 's with base $U$, where $|U|=\mu$, is denoted ${ }_{\mu} \mathrm{Cs}_{\alpha}$. A generalized cylindric field of sets is a $\mathrm{CA}_{\alpha}$ obtained by relativizing a set algebra to a unit set of the form $U_{i \in I}{ }^{\alpha} U_{i}$ where $\left\{U_{i}: i \in I\right\}$ is a collection of nonempty pairwise disjoint sets. The $U_{i}$ 's are called subbases and the class of such algebras is denoted by Gs ${ }_{\alpha}$. The class of $\mathrm{Gs}_{\alpha}$ 's where $\left|U_{i}\right|=\mu$ for each subbase $U_{i}$ is denoted ${ }_{\mu} \mathrm{Gs}_{\alpha}$. We are particularly interested in the case where $0<\mu, \alpha<\omega$. In this case, the class $I_{\mu} \mathrm{Gs}_{\alpha}$ is just the variety generated by $\mathfrak{A}(\mu, \alpha)$.

In a $\mathrm{CA}_{\alpha} \mathfrak{U}$ define

$$
C_{(\Gamma)} x=C_{k_{1}} \cdots C_{k_{n}}(x) \text { where } \Gamma=\left\{k_{1}, \ldots, k_{n}\right\} \subseteq \alpha .
$$

We also let

$$
d_{\Gamma}=\prod_{k, \lambda \in \Gamma} d_{k \lambda} \text { and } \bar{d}(\Gamma \times \Gamma)=\prod_{k, \lambda \in \Gamma: k \neq \lambda}-d_{k \lambda} \quad \text { for } \Gamma \subseteq \alpha .
$$

For short, let $\bar{d}=\bar{d}(\alpha \times \alpha)$.

A $\mathrm{CA}_{\alpha} \mathfrak{A}$ has characteristic $\mu, 0<\mu<\alpha \cap \omega$, if $\mathfrak{A}$ has a simple minimal subalgebra, $C_{(\mu+1)} \bar{d}((\mu+1) \times(\mu+1))=0$, and $C_{(\mu)} \bar{d}(\mu \times \mu)=1$. A CA F $_{\alpha}$, with a simple minimal subalgebra, has characteristic 0 if $C_{(\lambda)} \bar{d}(\lambda \times \lambda) \neq 1$ for all $\lambda<(\alpha+1) \cap \omega$. The class of $\mathrm{CA}_{\alpha}$ 's with characteristic $\mu$ is denoted ${ }_{\mu} \mathrm{CA}_{\alpha}$. For $0<\mu<\alpha<\omega,{ }_{\mu} \mathrm{CA}_{\alpha}=I_{\mu} \mathrm{Gs}_{\alpha}$ and all ${ }_{\mu} \mathrm{GS}_{\alpha}$ 's with $\mu \geqslant \alpha$ have characteristic 0 . The full set algebra $\mathfrak{A}(\alpha, \mu)$ is a simple algebra with characteristic $\mu$ if $0<\mu<\alpha<\omega$ and characteristic 0 if $\alpha \leqslant \mu<\omega$.

The set of all atoms of a $\mathrm{CA}_{\alpha} \mathfrak{B}$ is denoted $\mathrm{At}(\mathfrak{B})$. Atoms of simple minimal $\mathrm{CA}_{\alpha}$ 's have a nice description (see 2.4 .68 of [7]). For a simple minimal CA $\mathrm{A}_{\alpha} \mathfrak{A}$ of characteristic $\mu$ where $0 \leqslant \mu<\alpha<\omega$, the atoms of $\mathfrak{A}$ consist of all nonzero elements of the form $\bar{d}(\Gamma \times \Gamma) \cdot \prod_{\Delta \in P} d_{\Delta}$, where $P$ is a partition of $\alpha$ into $\leqslant \mu$ subsets if $\mu \neq 0$, and a partition of $\alpha$ if $\mu=0$, and $\Gamma$ is subset of $\alpha$ such that $|\Gamma \cap \Delta|=1$ for each $\Delta \in P$. The atom associated with a partition $P$ is denoted $a_{P}$.

The notion of a PEA $\alpha$ with characteristic $\mu$ is defined in the same way as for $\mathrm{CA}_{\alpha}$. An unpublished result of Leon Henkin states:

THEOREM 1.1. Every PEA $_{\alpha}, \alpha<\omega$, of positive characteristic is representable.

The next result shows that simple $\mathrm{PEA}_{\alpha}$ 's and $\mathrm{CA}_{\alpha}$ 's with positive characteristic are really the same. 
THEOREM 1.2. For $0<\mu<\alpha<\omega$ substitution operators can be defined on a simple ${ }_{\mu} \mathrm{CA}_{\alpha} \mathfrak{B}$ such that $\mathfrak{B}$ becomes a simple $\mathrm{PEA}_{\alpha}$ with characteristic $\mu$.

The proof of 1.2, which is quite long, will be published separately. The following corollary of 1.1 and 1.2 due to Leon Henkin will be used in 3.8 to help show that certain algebras are injective.

COROLLARY 1.3. Every ${ }_{\mu} \mathrm{CA}_{\alpha}$, with $0<\mu<\alpha<\omega$, is representable.

It follows from 1.3 that every simple ${ }_{\mu} \mathrm{CA}_{\alpha}, \mu>0$, is embeddable in $\mathfrak{A}(\alpha, \mu)$. The substitution operation on $\mathfrak{A}(\alpha, \mu)$ will be denoted $S(\tau)$, i.e., for $\tau \in{ }^{\alpha} \alpha$ and $x \in$ $\mathfrak{A}(\boldsymbol{\alpha}, \boldsymbol{\mu})$

$$
S(\tau) x=\left\{f \in{ }^{\alpha} \mu: f \tau \in x\right\} .
$$

The notation $S(\tau)$ is used in place of the standard $S_{\tau}$ to avoid confusion with $S_{\mu}$ which denotes the group of all permutations of $\mu$.

2. Galois theory. For $0<\mu, \alpha<\omega$ and a group $G$ of permutations on $\mu$, a subalgebra $\mathfrak{A}(\alpha, \mu)_{G}$ of $\mathfrak{A}(\alpha, \mu)$ is associated with $G$ in the following way. Let $\tilde{G}$ consist of all $\tilde{\sigma}$ for $\sigma \in G$, where $\tilde{\sigma}$ is the automorphism of $\mathfrak{A}(\alpha, \mu)$ defined for each $X \subseteq{ }^{\alpha} \mu$ by $\tilde{\sigma}(X)=\{\sigma y: y \in X\}$. It is easily seen that $\tilde{G} \subseteq$ Aut $\mathfrak{A}(\alpha, \mu)$ and the correspondence of $\sigma$ to $\tilde{\sigma}$ is an isomorphism $S_{\mu} \simeq$ Aut $\mathfrak{A}(\alpha, \mu)$. Let $\mathfrak{A}(\alpha, \mu)_{G}$ denote the subalgebra of $\mathfrak{A}(\alpha, \mu)$ whose universe consists of all elements fixed by every $\tilde{\boldsymbol{\sigma}} \in \tilde{\boldsymbol{G}}$.

For $\mathfrak{B} \subseteq \mathscr{A}(\alpha, \mu)$, the Galois group of $\mathfrak{B}$, denoted $G(\mathfrak{B})$, is the group of all $\sigma \in S_{\mu}$ such that $\tilde{\sigma}$ fixes each element of $\mathfrak{B}$.

The following result, whose proof is routine, connects subgroups of $S_{\mu}$ with subalgebras of $\mathfrak{A}(\alpha, \mu)$.

THEOREM 2.1. (1) If $H \subseteq G \subseteq S_{\mu}$, then $\mathfrak{A}(\alpha, \mu)_{G} \subseteq \mathfrak{A}(\alpha, \mu)_{H}$; moreover, $G \subseteq$ $G\left(\mathfrak{A}(\alpha, \mu)_{G}\right)$.

(2) If $\mathfrak{B} \subseteq \mathfrak{E} \subseteq \mathfrak{A}(\alpha, \mu)$, then $G(\mathfrak{E}) \subseteq G(\mathfrak{B})$; moreover, $\mathfrak{B} \subseteq \mathfrak{A}(\alpha, \mu)_{G(\mathfrak{B})}$.

The main goal in this section (Theorem 2.9) is to show that under certain conditions the correspondence in 2.1 becomes a lattice anti-isomorphism. For $\mu \leqslant \alpha$ the proof is related to ideas of Krasner [10]; for $\mu=\alpha+1$, the proof is due to $H$. Andréka and I. Németi.

For $G$ a subgroup of $S_{\mu}$ and $p \in{ }^{\alpha} \mu$ let $p^{G}=\{\sigma p: \sigma \in G\}$ denote the orbit of $p$ under the action of $G$ on ${ }^{\alpha} \mu$. Clearly, $\operatorname{At}\left(\mathfrak{A}(\alpha, \mu)_{G}\right)=\left\{p^{G}: p \in{ }^{\alpha} \mu\right\}$.

THEOREM 2.2. For $0<\mu \leqslant \alpha+1<\omega$ and a subgroup $G$ of $S_{\mu}, G=G\left(\mathfrak{A}(\alpha, \mu)_{G}\right)$; thus, the assignment of $\mathfrak{A}(\alpha, \mu)_{G}$ to $G$ is one-one.

Proof. The conclusion is trivial if $0<\mu \leqslant 2$; so assume that $2<\mu$ and $G \subseteq S_{\mu}$. By 2.1(1), $G \subseteq G\left(\mathfrak{A}(\alpha, \mu)_{G}\right)$. In case $\mu \leqslant \alpha$ choose $p \in{ }^{\alpha} \mu$ such that $\operatorname{Rg} p=\mu$. For $\sigma \in G\left(\mathfrak{A}(\alpha, \mu)_{G}\right), \tilde{\sigma}\left(p^{G}\right)=p^{G}$ so $\sigma p=\tau p$ for some $\tau \in G$. The choice $p$ implies $\sigma=\tau \in G$ and so $G=G\left(\mathfrak{A}(\alpha, \mu)_{G}\right)$, as desired. Now, assume $\mu=\alpha+1$ and $\sigma \in G\left(\mathfrak{A}(\alpha, \mu)_{G}\right)$. Choose $p \in{ }^{\alpha} \mu$ to be one-one and, as above, find $\tau \in G$ with $\sigma p=\tau p$. Since $\sigma$ and $\tau$ agree on $\operatorname{Rg} p$ and $\mu=|\operatorname{Rg} p|+1$, the permutations $\sigma$ and $\tau$ agree everywhere. Hence $\sigma=\tau \in G\left(\mathfrak{A}(\alpha, \mu)_{G}\right)$ as desired. 
H. Andréka and I. Németi have shown that Theorem 2.2 is the best possible in the sense that if $\mu \geqslant \alpha+2 \geqslant 4$, there exist distinct subgroups of $S_{\mu}$ with the same algebra of fixed points. For example, both $S_{\mu}$ and the alternating group on $\mu$ have the minimal subalgebra of $\mathfrak{A}(\alpha, \mu)$ as their algebra of fixed points since both are $\alpha$-fold transitive (because $\mu \geqslant \alpha+2$ ).

A function $f \in{ }^{\alpha} \mu$ is called normal if $\mu \leqslant \alpha$ and $\mu=\operatorname{Rg} f$ or if $\mu=\alpha+1$ and $f$ is one-one.

For $f \in{ }^{\alpha} \mu, i \in \alpha$, and $u \in \mu$ define $f(i / u)=\left(f \sim\left\{\left(i, f_{i}\right)\right\}\right) \cup\{(i, u)\}$. For $\mu=$ $\alpha+1$ and $f \in{ }^{\alpha} \mu$ normal, define $m_{i} f=f(i / u)$, where $u$ is the unique element of $\mu \sim \operatorname{Rg} f$.

The following facts about functions will be useful.

Lemma 2.3. Suppose $\mu=\alpha+1$ and $f \in{ }^{\alpha} \mu$ is normal. Then

(1) for each $i<\alpha, m_{i} m_{i} f=f$;

(2) for $\sigma \in S_{\mu}$ and $i<\alpha, m_{i}(\sigma f)=\sigma m_{i} f$;

(3) for $i<j<\alpha, f\left(i / f_{j}\right)\left(j / f_{i}\right)=m_{i} m_{j} m_{i} f$ so $S((i, j))\{f\}=\left\{m_{i} m_{j} m_{i} f\right\}$;

(4) for $g \in^{\alpha} \mu, g$ normal, there exists a finite sequence $i_{0}, \ldots, i_{n}$ of elements of $\alpha$ such that $m_{i_{0}} \cdots m_{i_{n}} f=g$.

Proof. (1)-(3) are clear.

(4) First suppose $\operatorname{Rg} f \neq \operatorname{Rg} g$, say $u \in \mu \sim \operatorname{Rg} f$ and $g_{i}=u$ for some $i \in \alpha$. Then $\operatorname{Rg} f=\operatorname{Rg} m_{i} g$ so it remains to consider $\operatorname{Rg} f=\operatorname{Rg} g$. In this case there is a permutation $\tau \in S_{\alpha}$ such that $g=f \tau$, i.e., $g \in S\left(\tau^{-1}\right)\{f\}$. Since $\tau^{-1}$ is a product of transpositions, the description of $g$ follows from (3).

For a subalgebra $\mathfrak{B}$ of $\mathfrak{A}(\alpha, \mu)$ and $f \in{ }^{\alpha} \mu$, let $X_{f}^{\mathfrak{B}}=\Pi\{b \in B: f \in b\}$. When the algebra $\mathfrak{B}$ is clear from the context we write $X_{f}$ for short. The $X_{f}$ 's are clearly the atoms of $\mathfrak{B}$.

LEMMA 2.4. For $\alpha, \mu<\omega, f \in{ }^{\alpha} \mu, i<\alpha$ and $\mathfrak{B} \subseteq \mathfrak{A}(\alpha, \mu)$ :

(1) $C_{i} X_{f}=\sum\left\{X_{q}: q \in C_{i}\{f\}\right\}$;

(2) for $\mu=\alpha+1$ and $f \in \bar{d}, m_{i} X_{f}=X_{m_{1} f}$.

Proof. (1) Since $f \in X_{f}, X_{q} \cdot C_{i} X_{f} \neq 0$ if $q \in C_{i}\{f\}$. Hence $X_{q} \leqslant C_{i} X_{f}$ because $X_{q}$ is an atom and so $C_{i} X_{f} \geqslant \sum\left\{X_{q}: q \in C_{i}\{f\}\right\}$. Now suppose $p \in C_{i} X_{f}$. Since $p(i / u) \in X_{f} \cdot C_{i} X_{p}$ for some $u, X_{f} \leqslant C_{i} X_{p}$. Thus, $f(i / v) \in X_{p}$ for some $v$. Let $q=f(i / v) \in C_{i}\{f\}$. Since $q \in X_{p}, p \in X_{p}=X_{q}$ from which the inclusion $\leqslant$ follows.

(2) For $f$ normal, $\bar{d} \cdot c_{i}\{f\}=\left\{f, m_{i} f\right\}$ so (1) implies $\bar{d} \cdot c_{i} X_{f}=X_{f}+X_{m_{i}} f$. If $X_{f}=\bar{d} \cdot c_{i} X_{f}$, then $X_{m_{i}} f=X_{f}$ and $m_{i} X_{f}=m_{i}\left(\bar{d} \cdot c_{i} X_{f}\right)=X_{f}$ from which $m_{i} X_{f}=$ $X_{m_{i} f}$ follows. If $\bar{d} \cdot c_{i} X_{f}$ is not an atom,

$$
X_{m_{l} f}=\bar{d} \cdot c_{i} X_{f} \cdot-X_{f} \leqslant m_{i} X_{f} .
$$

On the other hand,

$$
\bar{d} \cdot c_{i} X_{f}=\sum\left\{X_{q}+X_{m, q}: q \in X_{f}\right\}
$$


so

$$
\bar{d} \cdot c_{i} X_{f} \cdot-X_{f}=\sum\left\{X_{m_{i}}: q \in X_{f}\right\} \geqslant m_{i} X_{f},
$$

which gives $m_{i} X_{f}=X_{m_{i} f}$.

THEOREM 2.5. For $0<\mu \leqslant \alpha+1<\omega$ every subalgebra $\mathfrak{B}$ of $\mathfrak{A}(\alpha, \mu)$ is generated by a single element, namely, $\mathscr{B}=\operatorname{Sg}\left\{X_{p}\right\}$ where $p$ is normal.

Proof. See I.4.6 of [8] for the proof when $\mu \leqslant \alpha$. Assume $\mu=\alpha+1$. Since the atoms of $\mathfrak{B}$ have the form $X_{f}$ it suffices to show that if $p$ is normal, then $X_{f} \in \operatorname{Sg}\left\{X_{p}\right\}$ for every $f \in{ }^{\alpha} \mu$. From 2.4(1),

$$
d_{i j} \cdot c_{i} X_{f}=\sum\left\{x_{q}: q \in d_{i j} \cdot c_{i}\{f\}\right\}=X_{f(i / f j)}
$$

so that

$$
X_{f(i / f j)} \in \operatorname{Sg}\left\{X_{f}\right\} \quad \text { for every } f \in{ }^{\alpha} \mu .
$$

If $f$ is normal, the proof of $2.4(2)$ shows that $X_{m_{f} f}$ is either $X_{f}$ or $\bar{d} \cdot c_{i} X_{f} \cdot-X_{f}$. Hence $X_{m_{i} f} \in \operatorname{Sg}\left\{X_{f}\right\}$. By 2.3(3) it follows that

$$
X_{q} \in S g\left\{X_{f}\right\} \text { whenever } f \in \bar{d} \text { and } q=f\left(i / f_{j}\right)\left(j / f_{i}\right) .
$$

Since every $f \in{ }^{\alpha} \mu$ can be obtained from $p$ by a composition of transpositions and replacements, the desired conclusion follows from (1) and (2).

Theorem 2.5 was announced (without proof) for $\mu=\alpha+1$ in I.4.8 of [8] as the result $q(\alpha, \alpha+1)=1$. The exact relationship between $\mu$ and $\alpha$ for which the conclusion of 2.5 holds is still open. Some partial results are announced in Problem 8 of $[8$, p. 311].

LEMMA 2.6. Suppose $\mu \leqslant \alpha$ and $p, f \in \alpha \mu$ where $p$ is normal. Let $P=\left\{\Delta_{0}, \ldots, \Delta_{\mu-1}\right\}$ be the partition of $\alpha$, where $\Delta_{j}=p^{-1}\{j\}$ for $j<\mu$, and let $Q=\left\{\Gamma_{0}, \ldots, \Gamma_{\mu-1}\right\}$ be a similar partition obtained using $f$. Choose $\Delta, \Gamma \subseteq \alpha$ such that $\Delta \cap \Delta_{j}=\left\{v_{j}\right\}$ and $\Gamma \cap \Gamma_{j}=\left\{u_{j}\right\}$ for each $j \in \operatorname{Rg} f$. Let $\lambda_{f}$ denote a permutation of $\alpha$ such that $\lambda_{f}\left(u_{j}\right)=v_{j}$ for $j \in \operatorname{Rg} f$ and let $W=\lambda_{f}(\Gamma) \subseteq \Delta$. For $f \in a \in \operatorname{At}(\mathfrak{B})$, where $\mathfrak{B}$ is $a$ subalgebra of $\mathfrak{A}(\alpha, \mu)$, define $y_{f}=a_{P} \cdot C_{(\alpha-w)} S\left(\lambda_{f}\right) a$, where $a_{P}$ is the atom associated with the partition $P(c f . \S 1)$. Then $a_{Q} \cdot C_{(\alpha-\Gamma)} S\left(\lambda_{f}^{-1}\right) y_{f} \leqslant a$.

Proof. Suppose $q \in a_{Q} \cdot C_{(\alpha-\Gamma)} S\left(\lambda_{f}^{-1}\right) y_{f}$. Then there is $q^{\prime} \in S\left(\lambda_{f}^{-1}\right) y_{f}$ such that $q \uparrow \Gamma=q^{\prime} \uparrow \Gamma$ and for $q(i)=q\left(u_{j}\right)$ for all $i \in \Gamma_{j}$ (where $j \in \operatorname{Rg} f$ ). Since $q^{\prime}=h \lambda_{f}$ for some $h \in y_{f}$ there exists $h^{\prime} \in a$ such that $h^{\prime} \lambda_{f}^{-1} \uparrow W=h \uparrow W$. Now for $j \in \operatorname{Rg} f$,

$$
h^{\prime}\left(u_{j}\right)=h^{\prime} \lambda_{f}^{-1}\left(v_{j}\right)=h\left(v_{j}\right)=h \lambda_{f}\left(u_{j}\right)=q^{\prime}\left(u_{j}\right)=q\left(u_{j}\right) \text {. }
$$

Since $h^{\prime}, q \in a_{Q}, q=h^{\prime} \in a$, which establishes the inclusion.

Actually, $a_{Q} \cdot C_{(\alpha-\Gamma)} S\left(\lambda_{f}^{-1}\right) y_{f}=a$ holds in Lemma 2.6 but this is not needed below. In 2.8, Lemma 2.6 will be applied with $a=X_{p}$ where $p$ is normal. We will need to know that $y_{f} \in B$ whenever $f \in X_{p}$. For $\mu<\alpha$ we can appeal to 1.2 but a separate argument is needed for $\mu=\alpha$. It does not require much additional work to treat $\mu \leqslant \alpha$. As notation let $X \tau=\{f \tau: f \in X\}$ whenever $X \subseteq{ }^{\alpha} \mu$ and $\tau \in S_{\alpha}$. 
LEMMA 2.7. Suppose $0<\mu \leqslant \alpha<\omega, p \in{ }^{\alpha} \mu$ is normal, $\mathfrak{B}$ is a subalgebra of $\mathfrak{A}(\alpha, \mu)$ and $\tau \in S_{\alpha}$. Then

(1) $X_{p \tau}=X_{p} \tau$ belongs to $\operatorname{Sg}\left\{X_{p}\right\}$;

(2) $S(\tau) X_{p}$ is an atom of $\mathfrak{B}$.

Proof. (1) Since $\tau$ is a product of transpositions and, $f$ is normal iff $f \tau$ is normal, it suffices to consider only $\tau=(i, j)$ for $i, j<\alpha, i \neq j$. As in 2.6 associate a partition of $\alpha, P=\left\{\Delta_{0}, \ldots \Delta_{\mu-1}\right\}$, with $p$. If $p \in d_{i j}$, then $X_{p} \leqslant d_{i j}$, which yields $q \tau=q$ for all $q \in X_{p}$. It follows that $X_{p} \tau=X_{p}=X_{p \tau}$ in this case.

Now suppose $p \in-d_{i j}$ so that $i$ and $j$ belong to different blocks of the partition $P$. Without loss of generality, assume $i \in \Delta_{0}, j \in \Delta_{1}$ and denote $\Delta_{0}^{\prime}=\Delta_{0} \sim\{i\}$, $\Delta_{1}^{\prime}=\Delta_{1} \sim\{j\}$. Of course, either $\Delta_{0}^{\prime}$ or $\Delta_{1}^{\prime}$ (or both) may be empty. Consider the partition $R=\left\{\Delta_{0}^{\prime} \cup\{j\}, \Delta_{1}^{\prime} \cup\{i\}, \Delta_{2}, \ldots, \Delta_{\mu-1}\right\}$.

Case 1. $\Delta_{0}^{\prime}=\Delta_{1}^{\prime}=0$. Note that $P=R$ and $a_{R} \cdot c_{i} c_{j}\{p\}=\{p, p \tau\}$ so Lemma 2.4(1) yields

$$
\begin{aligned}
a_{R} \cdot c_{i} c_{j} X_{p} & =a_{R} \cdot \sum\left\{X_{q}: q \in c_{i} c_{j}\{p\}\right\} \\
& =\sum\left\{X_{q}: q \in a_{R} \cdot c_{i} c_{j}\{p\}\right\}=X_{p}+X_{p \tau}
\end{aligned}
$$

If $a_{R} \cdot c_{i} c_{j} X_{p}$ is an atom, then $X_{p}=a_{R} \cdot c_{i} c_{j} X_{p}=X_{p \tau}$, which yields

$$
X_{p} \tau=a_{R} \cdot\left\{q \tau: q \in c_{i} c_{j} X_{p}\right\}=a_{R} \cdot c_{i} c_{j} X_{p}=X_{p \tau}
$$

since $\left(c_{i} c_{j} X_{p}\right) \tau=c_{i} c_{j} X_{p}$. Also, note that $X_{p} \tau \in \operatorname{Sg}\left\{X_{p}\right\}$. Now, if $a_{R} \cdot c_{i} c_{j} X_{p}$ is not an atom, (3) yields

$$
X_{p \tau}=a_{R} \cdot c_{i} c_{j} X_{p} \cdot-X_{p}
$$

is in $\operatorname{Sg}\left\{X_{p}\right\}$. Also since $q \in X_{p}$ implies $X_{q}=X_{p}$, (3) yields

$$
a_{R} \cdot c_{i} c_{j} X_{p}=\sum\left\{X_{q}+X_{q \tau}: q \in X_{p}\right\}
$$

which gives

$$
a_{R} \cdot c_{i} c_{j} X_{p} \cdot-X_{p}=\sum\left\{X_{q \tau}: q \in X_{p}\right\} \geqslant X_{p}
$$

For $f \in a_{R} \cdot c_{i} c_{j} X_{p}, f \uparrow \alpha \sim\{i, j\}=g \uparrow \alpha \sim\{i, j\}$ for some $g \in X_{p}$ and $\mu=$ $\{f(i), f(j)\} \cup g^{*}(\alpha \sim\{i, j\})$. If $f \notin X_{p}, f \neq g$ so $f=g \tau \in X_{p} \tau$. Thus,

$$
X_{p \tau}=a_{R} \cdot c_{i} c_{j} X_{p} \cdot-X_{p}=X_{p} \tau
$$

as desired.

Case 2. $\Delta_{0}^{\prime} \neq 0$ or $\Delta_{1}^{\prime} \neq 0$. Assume, without loss of generality, that $k \in \Delta_{0}^{\prime}$. Suppose $f \in a_{R} \cdot c_{i} c_{j}\{g\}$, where $g \in a_{p}$. Then $f \uparrow \alpha \sim\{i, j\}=g \mid \alpha \sim\{i, j\}$ and $f(j)=f(k)=g(i)$. If $\Delta_{1}^{\prime} \neq 0$, say $l \in \Delta_{1}^{\prime}$, then $f(i)=f(l)=g$ so $f=g \tau$; while if $\Delta_{1}^{\prime}=0$, then $\mu=\{f(i)\} \cup g^{*}(\alpha \sim\{j\})$, which implies $f(i)=g(j)$, also yielding $f=g \tau$. Thus $a_{R} \cdot c_{i} c_{j}\{g\} \leqslant\{g \tau\}$. The argument above can be reversed to show

$$
a_{R} \cdot c_{i} c_{j}\{g\}=\{g \tau\}
$$


Applying (4) when $g \in X_{p}$ yields $a_{R} \cdot c_{i} c_{j} X_{p}=X_{p} \tau$. On the other hand, (4), with $g=p$, and $2.4(1)$ give

$$
a_{R} \cdot c_{i} c_{j} X_{p}=\sum\left\{X_{p}: q \in a_{R} \cdot c_{i} c_{j}\{p\}\right\}=X_{p \tau} .
$$

Thus, $X_{p \tau}=X_{p} \tau$ in Case 2 and part (1) follows.

(2) It is easily seen that $S(\tau) X_{p}=X_{p} \tau^{-1}$ which, by (1), is the atom $X_{p \tau^{-1}}$.

TheOREM 2.8. Suppose $0<\mu \leqslant \alpha+1<\omega$ and $\mathfrak{B}$ is a subalgebra of $\mathfrak{A}(\alpha, \mu)$. Let $G=G(\mathfrak{B})$ and $p \in^{\alpha} \mu$ be normal. Then $X_{p}=p^{G}$.

Proof. First assume that $\mu=\alpha+1$. Since $p \in X_{p}$ and $X_{p}$ is invariant under $G$, $p^{G} \subseteq X_{p}$. Now suppose $f \in X_{p}$. Then $f \in \bar{d}$ so we may choose $\alpha \in S_{\mu}$ with $f=\sigma p$. In order to show $\sigma \in G=G(\mathfrak{B})$ it suffices to show $\tilde{\sigma} X_{p} \subseteq X_{p}$ since, by $2.5, X_{p}$ generates $\mathfrak{B}$. For $q \in X_{p}, 2.3(4)$ gives $q=m_{i_{0}} \cdots m_{i_{n}} p$ (= $\bar{m} p$ for short) for some finite sequence $i_{0}, \ldots, i_{n}$ of elements of $\alpha$. Then $X_{p} \stackrel{n}{=} X_{q}=X_{\bar{m} p}=m X_{p}$ by 2.4(2). Hence $\sigma q=\sigma \bar{m} p=\bar{m} \sigma p=\bar{m} f \in \bar{m} X_{p}=X_{p}$ (using 2.3(2)) and, thus, $\sigma \in G$ as desired.

Now assume $\mu \leqslant \alpha$. Suppose $p, f \in{ }^{\alpha} \mu$ where $p$ is normal, and associate $P, Q, \Delta, \Gamma, u_{j}, v_{j}, \lambda_{f}$ and $W$ with $p$ and $f$ as in the hypothesis of Lemma 2.6. Note that

$$
p \uparrow W=f \lambda_{f}^{-1} \uparrow W .
$$

Hence $p \in y_{f}$ for every $f$. For the remainder of the proof we assume the atom $a$ in 2.6 is $X_{p}$ and $f \in X_{p}$. By $2.7(2), S\left(\lambda_{f}\right) X_{p}$ belongs to $\mathfrak{B}$ and, hence, each $y_{f} \in \mathfrak{B}$. It follows from (1) that $p \in y_{f}$ for each $f$, hence

$$
X_{p} \leqslant y_{f} \text { for each } f \in X_{p} .
$$

Now, for $\sigma \in S_{\mu}$,

$$
\sigma \notin G \text { implies } \sigma p \notin X_{p} .
$$

If $\sigma \notin G$, then $\tilde{\sigma}\left(X_{p}\right) \neq X_{p}$ by 2.5. Thus, $\sigma f \notin X_{p}$ for some $f \in X_{p}$. In view of (2), it suffices to show $\sigma p \notin y_{f}$. Because of $\in a_{Q}$ and $\sigma f \uparrow \Gamma=\sigma p \lambda_{f} \uparrow \Gamma$ by (1), it is not hard to see that

$$
\sigma f \in a_{Q} \cdot C_{(\alpha-\Gamma)} S\left(\lambda_{f}^{-1}\right)\{\sigma p\} .
$$

Now, if $\sigma p \in y_{f}$, (4) and 2.6 yield $\sigma f \in X_{p}$, which contradicts the choice of $f$. Hence (3) holds.

Finally, since $X_{p}$ is invariant under $G, p^{G} \leqslant X_{p}$. Now suppose $f \in X_{p} \leqslant a_{p}$. Then $P=Q$ and there exist $\sigma \in S_{\mu}$ such that $\Delta_{j}=\Gamma_{\sigma(j)}$ for $j<\mu$ and $f=\sigma p$. Since $\sigma p \in X_{p},(3)$ implies $\sigma \in G$. Therefore, $f \in p^{G}$ and, hence, $X_{p} \leqslant p^{G}$.

THEOREM 2.9. For $0<\mu \leqslant \alpha+1<\omega$ the correspondence in 2.1 between subgroups of $S_{\mu}$ and subalgebras of $\mathfrak{U}(\alpha, \mu)$ is a lattice anti-isomorphism.

ProOF. By 2.2 the correspondence is one-one and by 2.1 it is enough to show that, for every $\mathfrak{B} \subseteq \mathfrak{A}(\alpha, \mu), \mathfrak{B}=\mathfrak{A}(\alpha, \mu)_{G(\mathfrak{B})}$. Let $G=G(\mathfrak{B})$. By 2.1 we know that $\mathfrak{B} \subseteq \mathfrak{A}(\alpha, \mu)_{G}$ whenever $\mathfrak{B} \subseteq \mathfrak{U}(\alpha, \mu)$. By 2.5 and $2.8, \mathfrak{B}=\operatorname{Sg}\left\{p^{G}\right\}$ for any normal 
$p \in{ }^{\alpha} \mu$. However, the $p^{G}$ 's are atoms of $\mathfrak{A}(\alpha, \mu)_{G}$ and, by 2.5 , a normal $p \in{ }^{\alpha} \mu$ generates $\mathfrak{A}(\alpha, \mu)_{G}$. Thus, $\mathfrak{B}=\mathfrak{A}(\alpha, \mu)_{G}$ as desired.

It was noted following the proof of 2.2 that the assignment of $\mathscr{A}(\alpha, \mu)_{G}$ to $G$ was not one-one when $4 \leqslant \alpha+2 \leqslant \mu<\omega$. Thus, the correspondence in 2.9 will not be an anti-isomorphism in this case. However, one can also ask if, possibly, the correspondence could still be onto. The answer is negative. Andréka and Németi have produced an example of a set algebra $\mathfrak{B} \subseteq \mathfrak{A}(\alpha, \mu)$, again with $\alpha+2 \leqslant \mu<\omega$, for which $\mathfrak{R} \neq \mathfrak{A}(\alpha, \mu)_{G(\mathfrak{P})}$ and the minimal atom containing each normal $p$ is not an orbit. Thus, the assumption that $\mu \leqslant \alpha+1$ cannot be removed from either 2.8 or 2.9 .

3. Injectives and homogeneous algebras. For reasons that will become apparent in the next section it is useful to know the $\mathrm{CA}_{\alpha}$-injective algebras. For any class $K$ of similar algebras, an algebra $\mathfrak{A} \in K$ is a (weak) $K$-injective if for every $\mathfrak{H}$, $\mathfrak{S} \in K$, with $\mathfrak{B} \subseteq \mathbb{E}$, every (monomorphism) homomorphism $h: \mathfrak{B} \rightarrow \mathfrak{A}$ extends to a homomorphism $h^{+}$: $\mathfrak{S} \rightarrow \mathfrak{A}$.

By the following lemma we do not have to distinguish between weak $K$-injectives and $K$-injectives when $K$ is a homomorphism closed class of $\mathrm{CA}_{\alpha}$ 's.

LEMMA 3.1. For a class $K$ of similar algebras closed under homomorphic images and possessing the congruence extension property (CEP), an algebra is a $K$-injective if and only if it is a weak $K$-injective.

For many classes of algebras there are no nontrivial injective algebras, due to the following simple lemma.

LEMMA 3.2. For classes $K$ and $L$, with $K \subseteq L$, where all algebras in $K$ with more than one element have isomorphic minimal subalgebras, there are no nontrivial L-injective algebras in $K$ if $K$ contains simple algebras of arbitrarily high cardinality.

The lemma applies, in particular, to the case where $K$ is a variety, the notion of "simple algebra in $K$ " is elementary, and $K$ contains an infinite simple algebra. In the case of $\mathrm{CA}_{\alpha}$ 's,

COROllaRY 3.3. (i) There are no nontrivial injective $\mathrm{CA}_{1}$ 's.

(ii) For $\alpha>1$ there are no nontrivial injective $\mathrm{CA}_{\alpha}$ 's of characteristic 0 .

Proof. (i) Let $K=L=\mathrm{CA}_{1}$ in 3.2 and note that every BA can be made into a simple $\mathrm{CA}_{1}$. For (ii), if $1<\alpha<\omega$ it is not difficult to find an infinite simple algebra (and the notion of "simple algebra" is elementary). For $\alpha \geqslant \omega$, simple $L f_{\alpha}$ 's of arbitrarily high cardinality can be constructed from models.

Part (i) of 3.3 answers the specific part of Problem 4 in Halmos [5]. James S. Johnson observed that 3.2 also implies there are no injective modular lattices (cf. Balbes [1]). The following lemma is due to J. Donald Monk.

LEMMA 3.4. For $\alpha>1$ every complete discrete $\mathrm{CA}_{\alpha}$ is injective.

Proof. Since CEP holds in $\mathrm{CA}_{\alpha}$, by 3.1 it suffices to consider $\mathscr{B} \subseteq \mathbb{E}$ and a monomorphism $\mathfrak{B} \rightarrow \mathfrak{A}$ where $\mathfrak{A}$ is complete and discrete. If follows that $\mathfrak{B}$ and $\mathfrak{E}$ 
are discrete and, since the Boolean reduct of $\mathfrak{A}$ is injective, it follows that $\mathfrak{A}$ is injective as a $\mathrm{CA}_{\alpha}$.

From 3.4 there exist nontrivial $\mathrm{CA}_{\alpha}$-injectives for all $\alpha>1$. Below we will see there are nondiscrete ones for $\alpha<\omega$. The next two results reduce the question of injectives to each characteristic.

LEMmA 3.5. Suppose $a \mathrm{CA}_{\alpha} \mathfrak{A} \simeq P_{\lambda \in \Lambda} \mathfrak{A}_{\lambda}$ for some $\Lambda \subseteq \alpha \cap \omega$ and each $\mathfrak{A}_{\lambda}$ is $a$ nontrivial $\mathrm{CA}_{\alpha}$ with characteristic $\lambda$. Then $\mathfrak{A}$ is a $\mathrm{CA}_{\alpha}$-injective if and only if $\mathfrak{A}_{\lambda}$ is a $\mathrm{CA}_{\alpha}$-injective for all $\lambda \in \Lambda$.

Proof. Suppose $\mathfrak{A}$ is a $\mathrm{CA}_{\alpha}$-injective, $\lambda \in \Lambda, \mathfrak{B}$ and $\mathfrak{C}$ are $\mathrm{CA}_{\alpha}$ 's, $\mathfrak{R} \subseteq \mathfrak{C}$ and $h$ : $\mathfrak{B} \rightarrow \mathfrak{A}_{\lambda}$ is a homomorphism. Set $\mathfrak{B}_{\kappa}=\mathfrak{E}_{\kappa}=\mathfrak{A}_{\kappa}$ for $\kappa \in \Lambda, \kappa \neq \lambda$ and $\mathfrak{P}_{\lambda}=\mathfrak{R}$, $\mathfrak{C}_{\lambda}=\mathfrak{S}$. Then $P_{\kappa \in \Lambda} \mathfrak{B}_{\kappa} \subseteq P_{\kappa \in \Lambda} \mathfrak{E}_{\kappa}$ and $h$ induces a homomorphism $h^{+}: P_{\kappa \in \Lambda} \mathfrak{P}_{\kappa} \rightarrow \mathfrak{A}$. Since $\mathfrak{A}$ is $\mathrm{CA}_{\alpha}$-injective, $h^{+}$extends to a homomorphism $k^{+}: P_{\kappa \in \Lambda} \mathfrak{E}_{\kappa} \rightarrow \mathfrak{A}$. For $x \in P_{\kappa \in \Lambda} B_{\kappa}$ with $x_{\kappa}=0$ for $\kappa \neq \lambda$ and $x_{\lambda}=1, k^{+}$induces a homomorphism $k$ of $\mathfrak{C} \simeq\left(P_{\kappa \in \Lambda} \mathfrak{S}_{\kappa}\right) \uparrow x$ into $\mathfrak{A}_{\lambda}$ which extends $h$. Thus $\mathfrak{A}$ is a $C A_{\alpha}$-injective. The converse is obvious since a product of injectives is injective.

THEOREM 3.6. Every $\mathrm{CA}_{\alpha}$-injective algebra is a product of injectives of distinct characteristics.

Proof. A CA $\mathrm{CA}_{\alpha}$-injective is a retract of a complete $\mathrm{CA}_{\alpha}$ and thus complete. The theorem now follows from 3.5 using 2.4.66 and 2.1.33 of [7].

We now turn our attention to $\mathrm{CA}_{\alpha}$ 's with a fixed characteristic $\mu \neq 0$ and $\alpha<\omega$. A CA $\mathrm{CA}_{\alpha} \mathfrak{A}$ is called homogeneous if every isomorphism between subalgebras of $\mathfrak{A}$ extends to an automorphism of $\mathfrak{A}$.

As a consequence of the Galois theory result 2.9 there are natural homogeneous $\mathrm{CA}_{\alpha}$ 's. Similar results were obtained by Krasner [10] for another type of algebraic structure (cf. also [11, 12]).

THEOREM 3.7. For $0<\mu \leqslant \alpha+1<\omega, \mathfrak{A}(\alpha, \mu)$ is homogeneous.

Proof. Suppose $f: \mathfrak{B} \simeq \mathbb{E}$ where $\mathfrak{B}=\mathfrak{A}(\alpha, \mu)_{G}$ and $\mathfrak{E}=\mathfrak{H}(\alpha, \mu)_{H}$ according to 2.9. Define $p \in{ }^{\alpha} \mu$ by $p_{i}=\min \{i, \mu-1\}$ for $i<\alpha$. Clearly, $p$ is normal, $p^{G} \in \operatorname{At}\left(\mathcal{R}^{\mathbb{R}}\right)$, so $f\left(p^{G}\right) \in \operatorname{At}(\mathbb{E})$, say $f\left(p^{G}\right)=q^{H}$. Choose $\sigma \in^{\mu} \mu$ such that $\sigma(i)=q_{i}$ for $i<\mu \cap \alpha$ (and $\sigma(\alpha) \in \mu \sim \operatorname{Rg} q$ if $\mu=\alpha+1)$. Since $p^{G}, q^{H} \leqslant \bar{d}(\Gamma \times \Gamma)$, where $\Gamma=\alpha \cap \mu$, $\sigma$ is a permutation of $\mu$ and thus $\tilde{\sigma}$ is an automorphism of $\mathfrak{A}(\alpha, \mu)$ such that $\tilde{\boldsymbol{\sigma}}\left(p^{G}\right)=f\left(p^{G}\right)$. Since $\mathfrak{B}$ is generated by $\left\{p^{G}\right\}$ (see 2.5 and 2.8 ) it follows that $f$ is induced by $\tilde{\boldsymbol{\sigma}}$.

The next result shows there are $\mathrm{CA}_{\alpha}$-injectives with positive characteristic for $\alpha<\omega$. Recall that $I_{\mu} \mathrm{Gs}_{\alpha}$ is the subvariety of $\mathrm{CA}_{\alpha}$ generated by $\mathfrak{A}(\alpha, \mu)$.

THEOREM 3.8. (1) $\mathfrak{A}(\alpha, \mu)$ is $\mathrm{CA}_{\alpha}$-injective for $0<\mu<\alpha<\omega$.

(2) $\mathfrak{A}(\alpha, \mu)$ is an $I_{\mu} \mathrm{Gs}_{\alpha}$-injective for $0<\mu \leqslant \alpha+1<\omega$. 
Proof. (1) In view of 3.1 it suffices to consider

$$
\begin{aligned}
& \mathfrak{B} \\
& \text { UI } \\
& \mathfrak{A} \stackrel{f}{\rightarrow} \mathfrak{A}(\boldsymbol{\alpha}, \mu)
\end{aligned}
$$

where $\mathfrak{A}, \mathfrak{B}$ are $\mathrm{CA}_{\alpha}$ 's. Since $\mathfrak{A}(\alpha, \mu)$ has characteristic $\mu>0, \mathfrak{A}$ and $\mathfrak{B}$ satisfy the identity $C_{(\mu+1)} \bar{d}((\mu+1) \times(\mu+1))=0$ and, hence, we may assume $\mathfrak{A}$ and $\mathfrak{B}$ have characteristic $\mu$. For $I$ a maximal ideal of $\mathfrak{B}, \mathfrak{B} / I$ is a simple algebra of characteristic $\mu$, hence embeddable in $\mathfrak{A}(\alpha, \mu)$ by the representation result 1.3 .

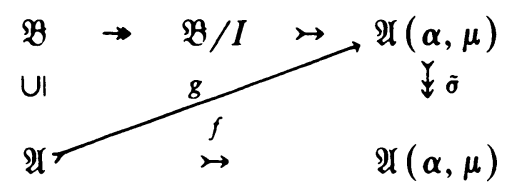

Let $g$ denote the composite homomorphism restricted to $\mathfrak{A}$. Use 3.7 to obtain an automorphism $\tilde{\sigma}$ of $\mathfrak{A}(\alpha, \mu)$ extending $f g^{-1}$. The outer diagram commutes giving a homomorphism that extends $f$.

(2) It suffices to consider $\mathfrak{A}, \mathfrak{B} \in I_{\mu} \mathrm{Gs}_{\alpha}$ such that

$$
\begin{aligned}
& \mathfrak{B} \\
& \stackrel{U}{f} \underset{f}{\mathfrak{A}} \mathfrak{A}(\boldsymbol{\alpha}, \boldsymbol{\mu})
\end{aligned}
$$

for some injection $f$. For any maximal ideal $I, \mathfrak{B} / I$ is simple. Because $\mathrm{CA}_{\alpha}$ 's have distributive congruence lattices, Jonsson's Lemma [9] implies that $\mathscr{B} / I$ is embeddable in $\mathfrak{A}(\alpha, \mu)$. The result now follows as in (1) using 3.7.

Theorem 3.8 yields several corollaries.

COROllaRy 3.9. For $1<\mu<\alpha<\omega, a \mathrm{CA}_{\alpha} \mathfrak{A}$ with characteristic $\mu$ is $\mathrm{CA}_{\alpha}$-injective if and only if $\mathfrak{U}$ is a retract of ${ }^{I} \mathfrak{A}(\alpha, \mu)$ for some $I \neq 0$.

An algebra with characteristic 1 is injective iff it is complete and discrete. All $\mathrm{CA}_{\alpha}$-injectives, for $\alpha<\omega$, can be described by combining 3.3(ii), 3.5, and 3.9.

The following will be needed in $\$ 4$.

Corollary 3.10. For $0<\mu \leqslant \alpha+1<\omega, I_{\mu} \mathrm{Gs}_{\alpha}$ has enough injectives, i.e., every member of $I_{\mu} \mathrm{Gs}_{\alpha}$ is embeddable in an $I_{\mu} \mathrm{Gs}_{\alpha}$-injective.

Using 2.4.66 of [7], for $\alpha<\omega$, a CA $\mathrm{CA}_{\alpha}$ that satisfies $\bar{d}(\alpha \times \alpha)=0$ is a product of $\mathrm{CA}_{\alpha}$ 's each with some positive characteristic. Thus,

COROllary 3.11. For $1<\alpha<\omega$, the variety of $\mathrm{CA}_{\alpha}$ 's defined by the equation $\bar{d}(\alpha \times \alpha)=0$ has enough injectives.

The hypothesis that $\mu \leqslant \alpha+1$ cannot be dropped from 3.7, 3.8 and 3.10. Andréka and Németi have shown that, for $\omega>\mu \geqslant \alpha+2, \mathfrak{A}(\alpha, \mu)$ is not homogeneous. It follows that the algebra is neither $I_{\mu} \mathrm{Gs}_{\alpha}$-injective nor can it be embedded in one. 
4. Amalgamation property. The amalgamation property for ${ }_{\mu} \mathrm{CA}_{\alpha}$ with $0<\mu<$ $\alpha \cap \omega$ was announced in [3]. Since ${ }_{\mu} \mathrm{CA}_{\alpha}=I_{\mu} \mathrm{Gs}_{\alpha}$ for $0<\mu<\alpha<\omega$, Theorem 4.2 will slightly improve the original result for $\alpha<\omega$.

The following easily proved lemma, due to R. S. Pierce, is a useful tool for establishing the amalgamation property.

LEMMA 4.1. Suppose $K$ is a class of similar algebras such that:

(i) $K$ is closed under isomorphisms and finite products;

(ii) $K$ has enough $K$-injectives.

Then $K$ has the amalgamation property.

The criteria 4.1 and 3.10 immediately yield:

THEOREM 4.2 (1) The amalgamation property holds in $I_{\mu} \mathrm{Gs}_{\alpha}$ for $0<\mu \leqslant \alpha+1<\omega$.

(2) The amalgamation property holds in ${ }_{\mu} \mathrm{CA}_{\alpha}$ for $0<\mu<\alpha<\omega$.

Tracing the arguments involved in the proof of 4.2(1) it is easy to see that for $\mu, \alpha<\omega, I_{\mu} \mathrm{Gs}_{\alpha}$ has the amalgamation property if and only if $\mathfrak{A}(\alpha, \mu)$ is homogeneous.

The restriction $\alpha<\omega$ in 4.2(2) can be removed using an ultraproduct argument involving reducts of $\mathrm{CA}_{\alpha}$ 's similar to the argument in Monk [13].

THEOREM 4.3. For $1<\mu<\omega,{ }_{\mu} \mathrm{CA}_{\alpha}$ has the amalgamation property for all $\alpha \geqslant \omega$.

Proof. Suppose $\alpha \geqslant \omega$ and $1<\mu<\omega$. Let $L$ be the set of all finite subsets $J$ of $\alpha$ such that $\mu+1 \subseteq J$, and for each $J \in L$ choose a one-one function $\gamma_{J}$ of $|J|$ onto $J$ that extends the identity on $\mu$. For $\mathfrak{A} \in \in_{\mu} \mathrm{CA}_{\alpha}$ let $\mathfrak{A}_{[J]}$ denote the $\gamma_{\mathcal{J}}$-reduct of $\mathfrak{A}$ (cf. 2.6.1 of [8]). Then $\mathfrak{A}_{[J]} \in_{\mu} \mathrm{CA}_{|J|}$ for $J \in L$.

Suppose $\mathfrak{A}, \mathfrak{B}$, $\mathfrak{夭}$ belong to ${ }_{\mu} \mathrm{A}_{\alpha}$ such that $\mathfrak{A} \subseteq \mathfrak{B}$ and $\mathfrak{A} \subseteq \mathfrak{E}$. By 4.2(2), the amalgamation property holds in ${ }_{\mu} \mathrm{CA}_{n}$ whenever $\mu<n<\omega$. Thus, for each $J \in L$, there exist a $\mathrm{CA}_{|J|} \mathscr{D}_{J}$ and monomorphisms $g_{J}, f_{J}$ such that the following diagram commutes ( $\delta$ is the identity embedding).

$$
\begin{array}{lll}
\mathfrak{B}_{[J]} & \stackrel{g_{J}}{\rightarrow} & \mathfrak{D}_{J} \\
\delta \hat{\imath} & & \hat{\imath} f_{J} \\
\mathfrak{A}_{[J]} & \stackrel{\delta}{\rightarrow} & \mathfrak{E}_{[J]}
\end{array}
$$

Let $M_{J}=\{K \in L: J \subseteq K\} \in \operatorname{Sb}(L)$ for $J \in L$. Then $M=\left\{M_{J}: J \in L\right\}$ generates a proper filter in $\operatorname{Sb}(L)$. Let $F$ denote an ultrafilter in $\operatorname{Sb}(L)$ that extends $M$.

A structure $D$ of the similarity type of $C A_{\alpha}$ can be defined as follows. We let the universe of $D$ equal the ultraproduct $P_{J \in L} D_{J} / F$ and define the Boolean operations in the usual way. For $\kappa, \lambda<\alpha$ define $d_{\kappa \lambda}^{\mathbb{D}}=h / F$, where $h \in P_{J \in L} D_{J}$ is defined by

$$
h(J)= \begin{cases}d_{i j}^{\mathbb{D}}, & \text { if } \kappa, \lambda \in J \in L, \\ \text { arbitrary } & \text { otherwise, }\end{cases}
$$

where $i=\gamma_{J}^{-1} \kappa$ and $j=\gamma_{J}^{-1} \lambda$. For $\kappa<\alpha$ and $h \in P_{J \in L} D_{J}$, define $c_{\kappa}(h / F)=k / F$, where $k \in P_{J \in L} D_{J}$ is defined so that when $\kappa \in J, k_{J}=c_{\gamma^{-1} \kappa}\left(h_{J}\right)$. 
The structure $\mathfrak{D}$ is a $\mathrm{CA}_{\alpha}$ with characteristic $\mu$. Define $g \in{ }^{B} D$ by $g(x)=h / F$, where $h \in P_{J \in L} D_{J}$ is defined by $h_{J}=g_{J} x$ for $J \in L$ and $x \in B$. Similarly, $f \in{ }^{C} D$ can be defined. Both $f$ and $g$ are monomorphisms and the diagram

$\begin{array}{ccc}\mathfrak{B} & \stackrel{g}{\hookrightarrow} & \mathfrak{D} \\ \delta \hat{\imath} & & \hat{\imath} f \\ \mathfrak{A} & \stackrel{\delta}{\hookrightarrow} & \mathfrak{E}\end{array}$

commutes.

The results above have the following corollary. Recall 2.4.66 of [7].

COROLLARY 4.4. The amalgamation property holds in the following classes:

(i) the class of $\mathrm{CA}_{\alpha}$ 's that satisfy $\bar{d}(\alpha \times \alpha)=0$ for $\alpha<\omega$; and

(ii) the class of $\mathrm{CA}_{\alpha}$ 's that satisfy $\bar{d}(\mu \times \mu)=0$ for $0<\mu<\alpha \cap \omega$.

In the next section we need to know that the amalgamation property holds for the class of simple algebras in various varieties. The next result allows us to apply the previous work.

THEOREM 4.5. The amalgamation property holds for the class of all simple algebras in a variety $V$ of $\mathrm{CA}_{\alpha}$ 's if the property holds in $V$.

Proof. Consider simple algebras $\mathfrak{A}, \mathfrak{B}, \mathfrak{S} \in V$ and monomorphisms $f: \mathfrak{A} \rightarrow \mathfrak{H}$ and $g: \mathfrak{A} \rightarrow \mathfrak{C}$. Suppose $\mathfrak{D}$ amalgamates $\mathfrak{A} \rightarrow \mathfrak{B}$ and $\mathfrak{A} \rightarrow \mathfrak{C}$ in $V$. Then, for a maximal ideal $I$ of $\mathfrak{D}, \mathfrak{D} / I$ is simple and it easily follows that $\mathfrak{D} / I$ also amalgamates $f: \mathfrak{A} \rightarrow \mathfrak{B}$ and $g: \mathfrak{A} \rightarrow \mathfrak{C}$.

5. Epimorphisms. In this section it will be shown that ES (that is, epimorphisms are surjective) holds for certain varieties of $\mathrm{CA}_{\alpha}$ 's. We assume throughout that $\alpha<\omega$.

It seems to be a folklore result that epimorphisms are onto in the case of Boolean algebras. An easy way to establish this is, via duality, to show that every monomorphism of Boolean spaces is a one-one map. The same strategy is employed in 5.3 for certain varieties of $\mathrm{CA}_{\alpha}$ 's. The appropriate dual of a $\mathrm{CA}_{\alpha}$ is the notion of a reduced $\alpha$-space introduced in [4]. For $\alpha<\omega$, a reduced $\alpha$-space is a sheaf $(X, \cong)$ of simple $\mathrm{CA}_{\alpha}$ 's over a Boolean space $X$. The duality between $\mathrm{CA}_{\alpha}$ 's and $\alpha$-spaces is reviewed in the next few paragraphs.

For a CA $\mathrm{CA}_{\alpha} \mathfrak{U}$ let $X(\mathfrak{U})$ denote the space of all maximal ideals of the $\mathrm{BA} \operatorname{Zd}(\mathfrak{A})$ of zero-dimensional elements of $\mathfrak{A}$. For $x \in X(\mathfrak{A}), \bar{x}=\{a \in A: a \leqslant z$ for some $z \in x\}$ is an ideal of $\mathfrak{A}$. The stalk $\mathfrak{S}_{x}$ over $x \in X(\mathfrak{A})$ is the $\mathrm{CA}_{\alpha} \mathfrak{A} / \bar{x}$ and $\mathfrak{S}(\mathfrak{U})=\bigcup\left\{\mathfrak{S}_{x}: x \in X(\mathfrak{U})\right\}$. For each $a \in A$ define $\sigma_{a}: X(\mathfrak{U}) \rightarrow \mathfrak{S}(\mathfrak{U})$ by $\sigma_{a}(x)=$ $a / \bar{x} . \mathfrak{S}(\mathfrak{U})$ is given the smallest topology which makes all $\sigma_{a}$ 's $(a \in A)$ open. Then $\mathfrak{A}^{d}=(X(\mathfrak{U}), \mathfrak{S}(\mathfrak{U}))$ is a reduced $\alpha$-space called the dual of $\mathfrak{A}$.

The construction of a $\mathrm{CA}_{\alpha}$ from a reduced $\alpha$-space uses the sectional functor. Let $\pi: \Im \rightarrow X$ denote the projection associated with an $\alpha$-space $(X, \Im)$. A function $\sigma$ : $X \rightarrow \Im$ is a section of $(X, \Im)$ if $\pi \sigma$ is the identity on $X$. The set $\Gamma(X, \subseteq)$ of all continuous sections of $(X, \Im)$ becomes a $\mathrm{CA}_{\alpha}$ by defining the operations pointwise. 
Of particular interest to the discussion here is the duality between $\mathrm{CA}_{\alpha}$ homomorphisms and sheaf morphisms. Given (reduced) $\alpha$-spaces $(Y, \cong)$ and $(X, \mathfrak{T})$, a sheaf morphism $H:(Y, \subseteq) \rightarrow(X, \mathfrak{T})$ is a pair $H=(\lambda, \mu)$, where $\lambda$ is a continuous map $Y \rightarrow X$ and $\mu$ is a continuous map $Y+{ }_{\lambda} \mathfrak{T} \rightarrow \cong$ such that $\mu_{y}=\mu(y,-)$ is a homomorphism of $\mathfrak{T}_{\lambda(y)}$ into $\mathfrak{S}_{y}$. We consider $Y+{ }_{\lambda} \mathfrak{T}=\{(y, t) \in Y \times \mathfrak{T}: \lambda(y)=$ $\pi t$ ) as a subspace of $Y \times \mathfrak{T}$. A sheaf morphism $(\lambda, \mu)=H:(Y, \cong) \rightarrow(X, \mathfrak{T})$ of $\alpha$-spaces produces a $\mathrm{CA}_{\alpha}$-homomorphism $\Gamma(H): \Gamma(X, \mathfrak{T}) \rightarrow \Gamma(Y, \Im)$ in the natural way: for $\sigma \in \Gamma(X, \mathfrak{I})$ define $\Gamma(H) \sigma$ by $(\Gamma(H) \sigma)(y)=\mu(y, \sigma(\lambda y))$ for all $y \in Y$. A sheaf morphism $h^{d}: \mathfrak{B}^{d} \rightarrow \mathfrak{A}^{d}$ can also be associated with a CA $\mathrm{CA}_{\alpha}$ homomorphism $h: \mathfrak{A} \rightarrow \mathfrak{B}$. Define $h^{d}=\left(h^{*}, h^{0}\right)$, where, for $y \in X(\mathfrak{B}), h^{*}(y)=h^{-1} \cap \operatorname{Zd}(\mathfrak{A})$ and, for $y \in X(\mathfrak{B})$ and $a \in A$,

$$
h^{0}\left(h, a / \overline{h^{*}(y)}\right)=h(a) / \bar{y} .
$$

Theorem 1.2 of [4] establishes a dual equivalence between $\mathrm{CA}_{\alpha}$ 's (with homomorphisms) and reduced $\alpha$-spaces (with sheaf morphisms). The next lemma describes sheaf morphisms dual to surjections of $\mathrm{CA}_{\alpha}$ 's.

Lemma 5.1. Suppose $\mathfrak{A}, \mathfrak{B} \in \mathrm{CA}_{\alpha}$ for $\alpha<\omega, h: \mathfrak{A} \rightarrow \mathfrak{B}$, and $h^{d}=\left(h^{*}, h^{0}\right)$ maps $\mathfrak{B}^{d} \rightarrow \mathfrak{A}^{d}$. Then $h$ is onto $\mathfrak{B}$ iff (i) $h^{*}$ is one-one, and (ii) for each $y \in X(\mathfrak{B}), h^{0}(y,-)$ is a surjection of the stalk over $h^{*}(y)$ onto the stalk over $y$.

Proof. Assume that (i) and (ii) hold. To simplity notation let $\mathfrak{A}^{d}=(X, \mathfrak{T})$ and $\mathfrak{B}^{d}=(Y, \subseteq)$. Because of the natural isomorphisms $\mathfrak{A} \simeq \Gamma\left(\mathfrak{A}^{d}\right)$ and $\mathfrak{B} \simeq \Gamma\left(\mathfrak{B}^{d}\right)$, to show that $h$ is onto it suffices to show that $\Gamma\left(h^{d}\right)$ is onto. Suppose $\sigma \in \Gamma(Y, \cong)$. For each $x \in Y, h^{0}(x,-)$ is onto so $h^{0}(x, t)=\sigma(x)$ for some $t \in \mathfrak{I}_{h^{*}(x)}$. In fact $t=\tau_{x}\left(h^{*}(x)\right)$ for some $\tau_{x} \in \Gamma(X, \mathfrak{T})$. Thus, there is a clopen neighborhood $N_{x}$ of $x$ such that $\Gamma\left(h^{d}\right)\left(\tau_{x}\right)(y)=\sigma(y)$ for all $y \in N_{x}$. The fact that $h^{*}$ is one-one and $X, Y$ are Boolean spaces implies that $h^{*}\left(N_{x}\right)$ is clopen in $h^{*}(Y)$ and there is a clopen set $M_{x}$ in $X$ such that $h^{*}\left(N_{x}\right)=M_{x} \cap h^{*}(Y)$. Using compactness (or the partition property of Pierce [15]) there exist a partition of $X$ into clopen subsets $M_{0}, \ldots, M_{k-1}$ and sections $\tau_{i} \in \Gamma\left(M_{i}, \mathfrak{T}\right)$ such that

$$
h^{0}\left(y, \tau_{i}\left(h^{*} y\right)\right)=\sigma(y)
$$

wherever $h^{*} y \in M_{i}$ for $i<k$. Defining $\tau$ by $\tau(z)=\tau_{i}(z)$ whenever $z \in M_{i}, i<k$, it follows that $\tau \in \Gamma(X, \mathfrak{T})$ and $\Gamma\left(h^{d}\right) \tau=\sigma$. Thus, $\Gamma\left(h^{d}\right)$ is onto $\Gamma\left(\mathfrak{B}^{d}\right)$ as desired. To prove the converse, first observe that a surjection $h$, restricted to closed elements, maps $\mathrm{Zd}(\mathfrak{A})$ onto $\mathrm{Zd}(\mathfrak{B})$ whenever $\alpha<\omega$. Thus, (i) follows from BA duality [6, p. 85] while (ii) follows from the definition of $h^{0}$.

When the hypothesis $\alpha<\omega$ is removed from 5.1, conditions (i) and (ii) no longer characterize an arbitrary surjection. In general, (i) and (ii) characterize conformal surjections, i.e., surjections $h: \mathfrak{A} \rightarrow \mathfrak{B}$ such that $h(\mathrm{Zd}(\mathfrak{A}))=\operatorname{Zd}(\mathfrak{A})$. If a $\mathrm{CA}_{\alpha} \mathfrak{A}$ is regular (in the sense of [4]) every surjection of $\mathfrak{A}$ onto $\mathfrak{B}$ is conformal. In particular, every $L f_{\alpha}$ is regular. We leave the details of the more general form of 5.1 to the reader.

The next result shows that "epis are onto" for certain classes of simple CA $\mathrm{A}_{\alpha}$ 's. 
LEMMA 5.2. The property ES holds in the following classes:

(1) simple $\mathrm{CA}_{1}$ 's;

(2) ${ }_{\mu} \mathrm{Cs}_{\alpha}$ for $0<\mu \leqslant \alpha+1<\omega$.

Proof. (1) Simple monadic algebras are Boolean algebras with a closure operator $c$ defined by $c x=1$ if $x \neq 0$ and $c 0=0$; thus the conclusion follows from the ES property for BA's.

(2) In order to show that every epimorphism between members of ${ }_{\mu} \mathrm{Cs}_{\alpha}$ is a surjection, it suffices to consider only inclusion embeddings. Assume $\mathfrak{A} \subset \mathfrak{B} \subseteq$ $\mathfrak{U}(\alpha, \mu)$. By the assumptions on $\mu$ and $\alpha$ the Galois correspondence 2.9 gives $\mathfrak{A}=\mathfrak{A}(\alpha, \mu)_{G}$ and $\mathfrak{P}=\mathfrak{A}(\alpha, \mu)_{H}$, where $H \subset G$. Thus, there exists an automorphism $\tilde{\sigma}$ of $\mathfrak{A}(\alpha, \mu)$ that fixes each $a \in \mathfrak{A}$ but moves some element of $\mathfrak{B}$. Since $\tilde{\sigma}$ and $\delta(=$ identity) agree on $\mathfrak{A}$ but differ on $\mathfrak{B}$, the inclusion is not an epimorphism whenever $\mathfrak{A}$ is a proper subalgebra of $\mathcal{B}$.

The main result of this section is presented next.

THEOREM 5.3. Suppose $V$ is a variety of $\mathrm{CA}_{\alpha}$ 's, $\alpha<\omega$, such that AP and ES hold in the class of simple members of $V$. Then ES holds in $V$.

Proof. In view of the duality results [4] and Lemma 5.1, it suffices to show that every monomorphism in the category of reduced $\alpha$-spaces dual to $V$ satisfies properties 5.1(i) and 5.1(ii). We assume $(X, \mathfrak{I})$ and $(Y, \Xi)$ are sheaves of simple algebras in $V$ over Boolean spaces $X$ and $Y$ and $(h, k)=H:(Y, \Xi) \rightarrow(X, \mathfrak{I})$ is a monomorphism.

Suppose $h x=h y$ for some $x, y \in Y$. Since $\Xi_{x}, \Xi_{y}$, and $\mathfrak{I}_{h x}$ are simple algebras in $V$, the amalgamation property implies there exist a simple $\mathfrak{I}$ in $V$ and monomorphisms $f_{x}: \Im_{x} \rightarrow \mathfrak{I}$ and $f_{y}: \widetilde{\Xi}_{y} \rightarrow \mathfrak{I}$ such that

$$
f_{x} \circ k_{x}=f_{y} \circ k_{y} .
$$

Consider the sheaf $(1, \mathfrak{L})$ over the one-point space $\{0\}=1$ and sheaf morphisms

$$
H_{x}=\left(\lambda_{x}, \mu\right):(1, \mathfrak{I}) \rightarrow(Y, \cong) \text { and } H_{y}=\left(\lambda_{y}, \nu\right):(1, \mathfrak{I}) \rightarrow(Y, \cong),
$$

where $\lambda_{x}(0)=x, \lambda_{y}(0)=y, \mu_{0}=f_{x}$, and $\nu_{0}=f_{y}$. The sheaf $(1, \mathfrak{I})$ is the $\alpha$-space dual to $\mathfrak{D} \in V$ and (1) implies that $H \circ H_{x}=H \circ H_{1}$. Since $H$ is a monomorphism, $H_{x}=H_{y}$, from which $x=y$. Thus, $h$ is one-one and 5.1(i) holds.

Fix $x \in Y$. Since ES holds for the simple algebras of $V$, in order to show that $k_{x}$ : $\mathfrak{I}_{h x} \rightarrow \mathfrak{S}_{x}$ is onto, it suffices to show that $k_{x}$ is an epi. Hence, suppose $f_{0}: \mathfrak{S}_{x} \rightarrow \mathfrak{D}$ and $f_{1}: \mathbb{S}_{x} \rightarrow \mathfrak{D}$ for some (simple) $\mathfrak{D}$ such that

$$
f_{0} \circ k_{x}=f_{1} \circ k_{x} \text {. }
$$

Introduce sheaf morphisms

$$
H_{0}=(\lambda, \mu):(1, \mathfrak{T}) \rightarrow(Y, \cong) \text { and } H_{1}=(\lambda, \nu):(1, \mathfrak{D}) \rightarrow(Y, \cong),
$$

where $\lambda(0)=x, \mu_{0}=f_{0}$ and $\nu_{0}=f_{1}$. From (2) it follows that $H \circ H_{0}=H \circ H_{1}$, but since $H$ is a monomorphism this yields $H_{0}=H_{1}$, from which $f_{0}=f_{1}$. Thus, we see that $k_{x}$ is an epi and hence maps onto $\mathbb{S}_{x}$. Since $x \in Y$ is arbitrary, 5.1(ii) holds. This completes the proof of 5.3. 
Some consequences of 5.3 are considered below.

\section{Corollary 5.4. ES holds in $\mathrm{CA}_{1}$.}

Proof. The amalgamation property for BA's implies AP for the class of simple $\mathrm{CA}_{1}$ 's. The result follows by $5.2(1)$ and 5.3.

The result 5.4 has also been established by $\mathrm{I}$. Sain, who has shown that $\mathrm{CA}_{1}$ satisfies the strong amalgamation property.

COROLlaRy 5.5. ES holds in $I_{\mu} \mathrm{Gs}_{\alpha}$ where $0<\mu \leqslant \alpha+1<\omega$. In particular, it holds in ${ }_{\mu} \mathrm{CA}_{\alpha}$ for $0<\mu<\alpha<\omega$.

Proof. By 5.3, 5.2(2), 4.5, and 4.2.

The following corollary was pointed out by H. Andréka and I. Németi.

COROLlaRY 5.6. ES holds in the subvariety of $\mathrm{CA}_{\alpha}$ 's defined by $\bar{d}(\alpha \times \alpha)=0$ for $\alpha<\omega$.

Proof. The simple algebras of this subvariety are just the simple algebras of characteristic $\mu, 0<\mu<\alpha$. Thus, ES for the simple algebras holds by 5.2 and AP holds by 4.4 and 4.5 .

In joint work with $H$. Andréka and I. Németi it has been shown that if $1<\alpha<\omega$, there exist epimorphisms in $\mathrm{CA}_{\alpha}$ which are not surjective. Moreover, 5.2(2) and 5.5 are best possible in the sense that ES fails in the variety $I_{\mu} \mathrm{Gs}_{\alpha}$ when $\omega>\mu>\alpha+1$.

\section{REFERENCES}

1. R. Balbes, Projective and injective distributive lattices, Notices Amer. Math. Soc. 13 (1966), 740.

2. S. Comer, Some representation theorems and the amalgamation property in algebraic logic, Ph.D. Thesis, Univ. of Colorado, 1967.

3. Galois theon and the amalgamation property in finite dimensional cylindric algehras, Notices Amer. Math. Soc. 15 (1968), 103.

4. __ A sheaf-theoretic duality theon for cylindric algebras, Trans. Amer. Math. Soc. 169 (1972). $75-87$.

5. P. R. Halmos, Injective and projective Boolean algebras, Lattice Theory, Proc. Sympos. Pure Math., vol. 2, Amer. Math. Soc., Providence, R. I., 1961, pp. 114-120.

6. B. Boolean algebras, Van Nostrand, 1963: reprinted, Springer-Verlag, 1974.

7. L. Henkin, J. D. Monk and A. Tarski, Cylindric algebras. Part I, North-Holland, 1971.

8. L. Henkin et al, Cylindric set algebras, Lecture Notes in Math., vol. 883. Springer-Verlag, 1981.

9. B. Jonsson, Algebras whose congruence lattices are distributive, Math. Scand. 21 (1967), 110-121.

10. M. Krasner, Une generalisation de la notion de corps, J. Math. Pures Appl. 17 (1938), 367-385.

11. Generalisation abstract de la theorie de Galois, Colloq. Internat. CNRS. No. 24. 1950, pp. $163-168$.

12. Les algèbres cylindriques, Bull Soc. Math. France 86 (1959), 315-319.

13. J. D. Monk, Model-theoretic methods and results in the theon of cylindric algebras, The Theory of Models (J. W. Addison et al., eds.), North-Holland, 1965, pp. 235-250.

14. I. Németi, Surjectiveness of epis is equitalent to Beth definability in algebraic logic, Budapest. 1982. (preprint)

15. R. S. Pierce, Modules over commutative regular rings, Mem. Amer. Math. Soc. No. 70 (1967).

16. D. Pigozzi, Amalgamation, congruence-extension, and interpolation properties in algehras. Algebra Universalis 1 (1972), 269-349.

Department of Mathematics and Computer Science. The Citadel. Charleston. South Carolina 29409 